\title{
COMBINING THEORY AND EXPERIMENT FOR UNDERSTANDING OF ULTRAFAST PHOTOINDUCED CHARGE-TRANSFER PROCESSES ${ }^{1}$
}

\author{
Eric Vauthey \\ Dr. es Sciences, Professor, Department of Physical Chemistry, \\ University of Geneva \\ eric.vauthey@unige.ch \\ Quai Ernest-Ansermet, 30, CH-1211 Genève 4, Switzerland
}

\begin{abstract}
This article gives a brief overview of the long-standing collaboration between our experimental spectroscopy group in Geneva and the theoretical group lead by Prof. A.I. Ivanov in Volgograd. This fruitful collaboration resulted in a significant increase of our understanding of the dynamics of several ultrafast charge-transfer processes in the condensed phase.
\end{abstract}

Key words: Charge separation and recombination, electron transfer symmetry breaking, time resolved spectroscopy, electron transfer theory, magnetic field effect.

\section{Introduction}

My research group in Geneva is interested in the dynamics occurring after optical excitation of molecules, mostly organic, in liquids $[14 ; 20]$. These dynamics are investigated using a combination of ultrafast time-resolved spectroscopic techniques including transient absorption in the UV, visible, near IR and mid IR regions as well as transient emission spectroscopy. The combination of this ensemble of spectroscopic data allows a comprehensive picture of the dynamics to be deduced. The obtained results are also used to put relevant theoretical models to the test. Before the advent of ultrafast spectroscopy, the dynamics of most photoinduced processes could be well discussed using conventional kinetic models with well-defined reaction intermediates and rate constants. However, thanks to the increasing time-resolution of laser spectroscopy, it is now possible to measure photoinduced reactions occurring on similar timescales as those of solvent motion and vibrational relaxation. As dis(2) cussed in more detail below, these reactions do no longer follow simple exponential kinetics. 
Moreover, their dynamics and efficiency may significantly depend on the excitation wavelength, breaking the famous Kasha-Vavilov rule of photochemistry, stating that the outcome of a photochemical reaction does not depend on the excitation wavelength [3]. Therefore, a proper description of these processes needs advanced theoretical modelling that accounts for the non-equilibrium nature of the dynamics. I became familiar with the work of A. Ivanov on the theoretical description of non-equilibrium charge-transfer processes in 2001 during the 20th edition of the International Conference on Photochemistry (ICP) in Moscow. This meeting with A. Ivanov was a happy coincidence as my group was precisely investigating non-equilibrium charge-transfer processes. This was the start of a fruitful collaboration that is still continuing now. Some of the outcomes resulting from this continuous collaboration between our two groups are described below. They concern both intermolecular and intramolecular photoinduced charge-transfer processes in liquids.

\section{Intermolecular photoinduced electron transfer}

An overall scheme of an bimolecular photoinduced electron transfer (ET) reaction is illustrated in Figure 1. In general, optical excitation leads to the population of the locally $S_{1}$ state of either the electron donor (D) or the electron acceptor (A). For charge separation (CS) to take place, the $\mathrm{D}$ and $\mathrm{A}$ reactants should be at a distance/orientation where the electronic coupling is sufficiently large. CS quenches the fluorescence of the excited reactant $\left(D^{*}\right.$ in Figure 1) and results in the formation of an ion pair. The latter can either recombine or separate into free ions. Free ions are the species of interest for most applications of these reactions, which include photopolymerisation, solar energy conversion and organic synthesis. A large free ion yield requires a slow charge recombination (CR) of the ion pairs relatively to their dissociation. Therefore, a deep understanding of the CR dynamics of ion pairs and of the parameters that control this process is crucial for the above-mentioned applications.

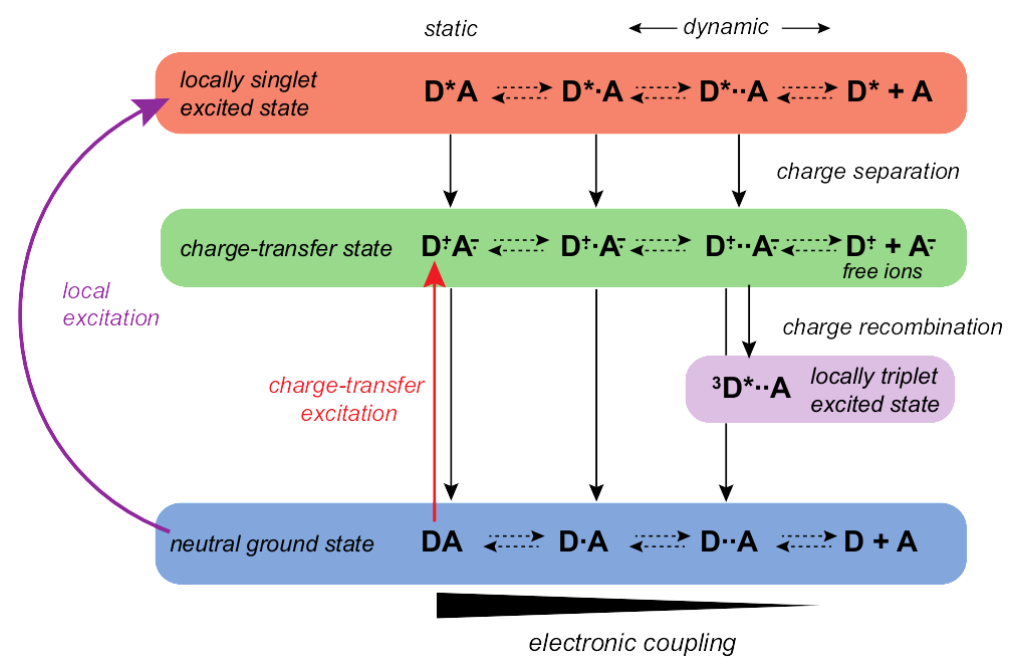

Fig. 1. Overall scheme of a bimolecular electron-transfer quenching process in polar solvents. The dashed arrows are used to stress the continuous distribution of pairs with different distances/orientations and coupling strengths

The investigation of ion-pair recombination is complicated by the fact that its dynamics is convolved with that of the ion-pair formation upon photoinduced CS. The latter depend 
strongly on the reactant-pair distribution, which is time dependent. Indeed, CS first takes place between reactant pairs that are at optimal distance/orientation. In this case, no diffusion is required and the fluorescence quenching is called static. After this subpopulation has reacted, diffusion is required to bring reactants at distances where CS is operative. In this limit, CS is usually diffusion controlled and the quenching is called dynamic. Because of the time-dependence of the reactant-pair distribution, CS quenching does not follow an exponential dynamics with a well-defined rate constant. Instead, the reaction rate varies with time as the quenching evolves from the static to the dynamic, diffusion-controlled, regime. Consequently, an adequate theoretical description of the CS dynamics requires a proper model that takes the time-dependent reactant-pair distribution into account $[1 ; 2]$. I will come back to such models later.

This complication can be avoided by investigating the $\mathrm{CR}$ of ion pairs generated upon direct charge-transfer excitation of DA complexes in the ground state (red arrow in Figure 1). These complexes consist of reactant pairs with sufficient molecular orbital overlap to allow direct optical transition to the ion-pair state. In this case, ion pairs are produced 'instantaneously' upon excitation and their recombination dynamics are not convolved with those of CS. Using visible transient absorption spectroscopy, we could measure the CR of several DA complexes and thus investigate the effect of driving force and solvent on the dynamics [19]. We first observed that the CR dynamics were not exponential and that their dependence on the driving force departed significantly from the prediction of Marcus ET theory $[15 ; 16]$, assuming instantaneous solvent response or fully solvent-controlled dynamics (Figure 2).

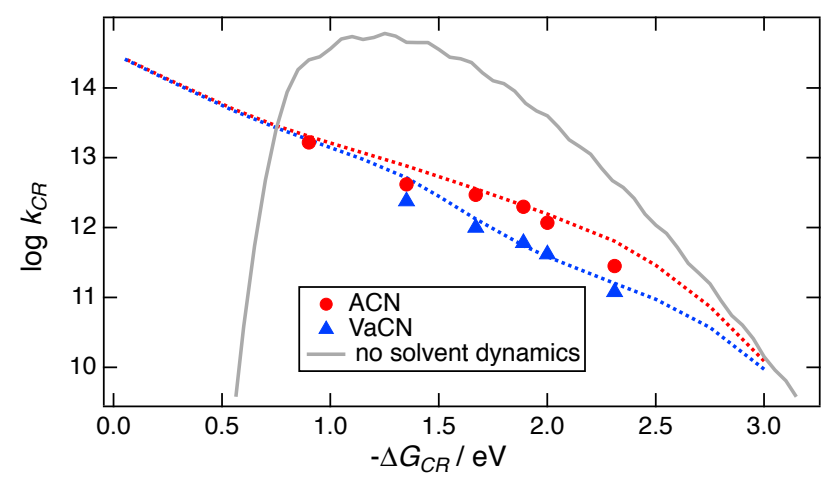

Fig. 2. Driving-force dependence of the $\mathrm{CR}$ rate constant measured with several excited DA complexes in acetonitrile $(\mathrm{ACN})$ and valeronitrile $(\mathrm{VaCN})$. The solid grey line is the prediction of Marcus theory assuming instantaneous solvent response. The dashed lines are simulations of a simple non-equilibrium dynamics model [19]

We started collaborating with the Ivanov group to better understand the origin of the non-exponential CR dynamics as well as its driving-force and solvent dependence. As we suspected non-equilibrium dynamics to be at play, we were wondering how its could depend on the excitation wavelength. Such an effect, called spectral effect, was first predicted using a perturbative theory approach [12]. It was later confirmed by simulations where wave-packet motion and electronic transition were described within the framework of the stochastic point-transition approach [6;7]. The CR dynamics were found to be significantly 
non-exponential and to slow down with increasing excitation energy, in excellent agreement with our measurements. Further theoretical investigations revealed that this spectral effect decreases with increasing electronic coupling [17].

Several DA complexes are characterised by two charge-transfer absorption bands corresponding to different mutual orientations of the $\mathrm{D}$ and $\mathrm{A}$ subunits. In this case again, the difference in $\mathrm{CR}$ upon excitation in the two bands could be well reproduced by the theoretical simulations [10]. The non-equilibrium nature of the CR dynamics of excited DA complexes and the spectral effect can be qualitatively understood using the simple free-energy parabola model depicted in Figure 3.

Non-equilibrium CR dynamics can also take place in ion pairs produced upon dynamic quenching. However, as mentioned above, the observed ion-pair decay is the convolution of the ion-pair production upon CS and the CR dynamics itself. According to Marcus ET theory $[15 ; 16]$, weakly exergonic CR should be relatively slow, favouring large free ion yields. However experimental measurements point to ultrafast recombination and, thus, negligibly small free ion yields. In a joint collaboration with A. Burshtein, one of the pioneers in the theory of diffusion-assisted reactions [1;2], and A. Ivanov, non-equilibrium CR dynamics was included in the so-called unified theory of CS/CR. It was then possible to reproduce the $\mathrm{CR}$ dynamics of ion pairs produced upon local excitation of the donor, including thus both static and dynamic quenching [9]. This example illustrates the importance of non-equilibrium dynamics in ultrafast electron-transfer processes. Its neglect can lead to totally wrong predictions on the rate of photoinduced charge-transfer processes and on the free ion yield.

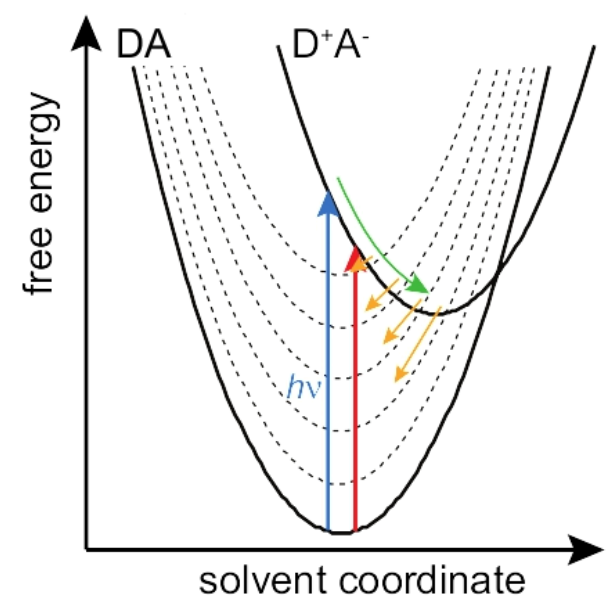

Fig. 3. Free energy of the ground and excited states of a donor-acceptor complex along the solvent coordinate. The dashed parabolas represent vibrational excited states of the neutral state, the green arrow the solvent relaxation, and the orange arrows the charge-recombination pathways. The probability of charge-recombination is represented by the length of the orange arrows. High-frequency excitation (blue arrow) prepares the excited ion-pair state further from equilibrium than low-frequency excitation (red arrow)

The CR dynamics of ion pairs can be influenced on a slower timescale by applying an external magnetic field. Depending on the nature of the DA pair, CR can either repopulate the neutral ground state or a local triplet excited state of the reactant pair (Figure 1). Spin 
conversion of the ion pair, initially produced in the singlet state, is required for triplet CR to be operative. It can take place via hyperfine interaction as soon as the two ions are at distances where the exchange interaction is sufficiently small for the singlet and triplet ion-pair states to be degenerate. This spin conversion can be slowed down by lifting the degeneracy of the triplet sub-levels with an external magnetic field. Using ps-ns transient absorption, we measured the time evolution of the triplet population arising from triplet recombination and observed a decrease of about $15 \%$ of the triplet yield in the presence of a magnetic field [8]. This effect was smaller than expected assuming a conventional description of spin conversion. However, the Ivanov group was able to reproduce both the time evolution of the triplet population and the magnetic field effect using a diffusion-assisted reaction model with the spin conversion described as a coherent process [8]. This result gives a new insight into the coupling of ion pairs on their way to free ions.

\section{Intramolecular photoinduced charge transfer}

Over the past few years, my group has been interested in symmetry-breaking chargetransfer processes $[4 ; 22]$. Two types of such processes can be distinguished: the first is a photoinduced charge separation between two identical molecules. It offers promising perspectives for solar energy conversion. The second, called excited-state symmetry breaking (ES-SB), takes place in multi-branched DA molecules. Symmetric two- or three-branched multipolar molecules are currently attracting strong interest, because they usually exhibit a high two-photon absorption cross-section. This property is very useful for a broad range of applications including fluorescence imaging, photopolymerisation, phototherapy and optical data storage. ES-SB is schematised in Figure 4 left. Directly after photoabsorption, electronic excitation is spread evenly over the molecule and the excited state is quadrupolar. Upon ES-SB, excitation localises on one of the two branches and the excited state becomes dipolar. This phenomenon was first identified by the strong solvent dependence of the fluorescence spectrum of centro-symmetric molecules, that pointed to a strongly dipolar excited state. ES-SB due to thermal fluctuations of the molecular structure and/or of the environment was invoked to account for this observation [21].
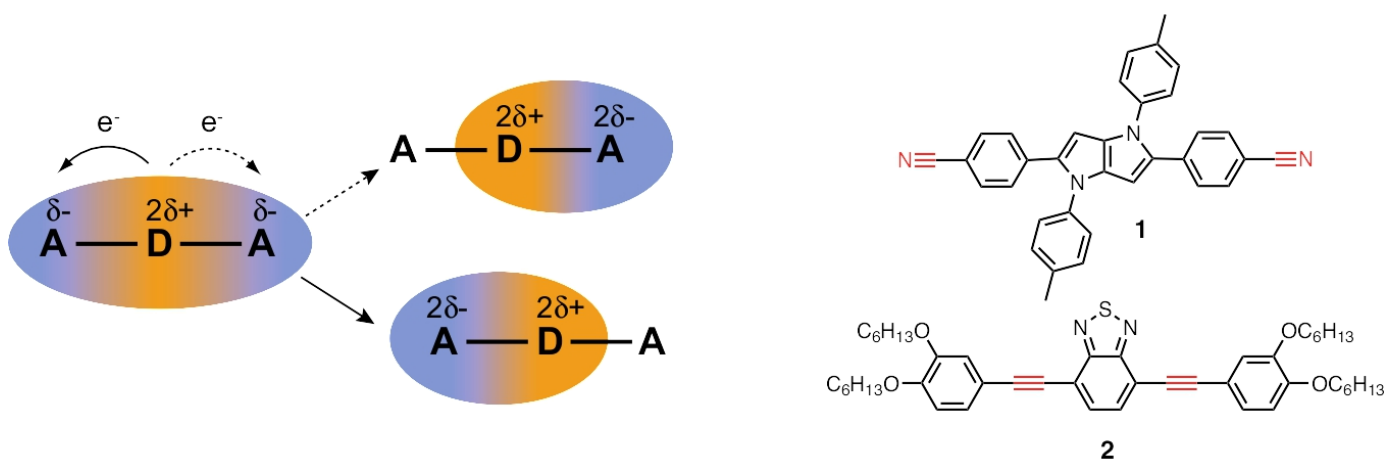

Fig. 4. Left: schematic representation of excited-state symmetry breaking (ES-SB) in a two-branched DA molecule. Directly after absorption of light, electronic excitation is distributed evenly over the molecule and the excited state is quadrupolar. Upon ES-SB, excitation localises on one branch leading to a dipolar excited state. Right: example of $A-\pi-D-\pi-A(\mathbf{1})$ and $D-\pi-A-\pi-D(2)$ molecules which were used to visualise ES-SB by time-resolved vibrational spectroscopy. The vibrational modes that were probed are shown in red

ISSN 2587-6325. Математ. физика и компьютер. моделирование. 2020. T. 23. № 2 
For many years, several experimental groups have been trying without success to observe ES-SB using transient electronic spectroscopy. The measured transient electronic spectra did not exhibit any clear spectral signature of this phenomenon. Vibrational spectroscopy is well-known for its high sensitivity to symmetry. Therefore, a few years ago we embarked into time-resolved IR (TRIR) studies of ES-SB [4]. For this, we selected symmetric molecules with $D-\pi-A-\pi$-D and $A-\pi-D-\pi$-A motifs, where $-\pi-$ is a $\pi$ conjugated linker, and we monitored vibrations localised either in the $\pi$ linker or at the D or A end of the branches (Figure 4, right). In principle, symmetric molecules like $\mathbf{1}$ and $\mathbf{2}$ have two $-C \equiv N$ or $-C \equiv C-$ stretching vibrations: a Raman-active but IR-inactive symmetric one, and a Raman-inactive but IR-active antisymmetric stretching mode. If symmetry is broken, both vibrations are visible using either Raman or IR spectroscopy. Figure 5A reveals that after $S_{1} \leftarrow S_{0}$ excitation of dye $\mathbf{1}$ in a non-polar solvent, the IR spectrum exhibits a single $-C \equiv N$ stretching band that remains unchanged during the entire excited-state lifetime. This indicates that, in this solvent, the $S_{1}$ state of $\mathbf{1}$ is symmetric and purely quadrupolar. However, the same measurements in polar solvents show that, after a few picoseconds, the band is split into an intense and a weak $-C \equiv N$ stretching bands. This is a direct effect of ES-SB, after which the 'symmetric' $-C \equiv N$ vibration is no longer totally IR-inactive and appears as a weak IR band [5]. An alternative way to explain this effect is that the electronic density on both arms is no longer the same and, thus, the $-C \equiv N$ stretching frequencies on both branches differ.

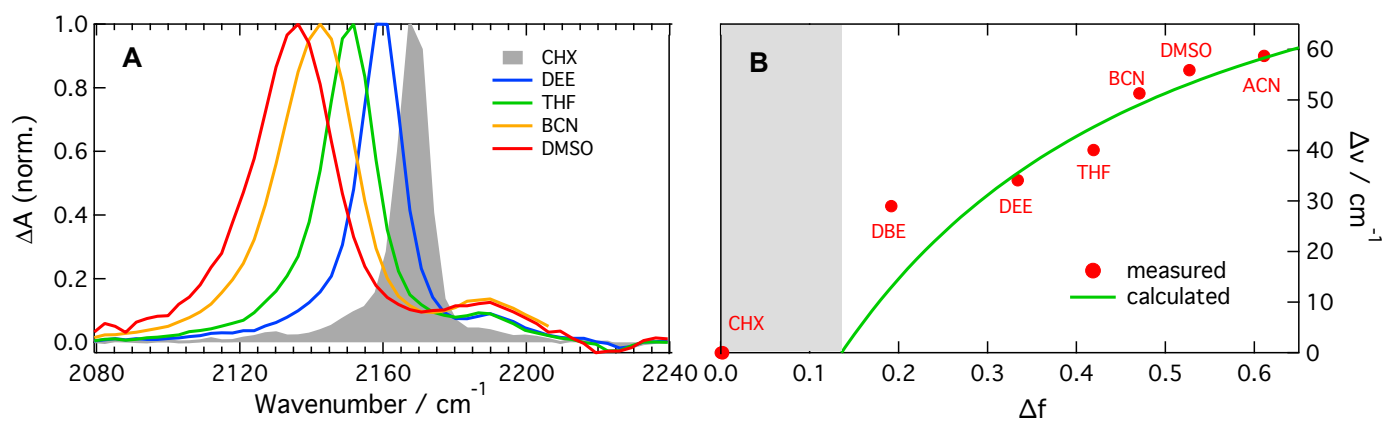

Fig. 5. A) Transient IR spectra recorded a few picoseconds after excitation of dye $\mathbf{1}$ in solvents of varying polarity. B) Experimental frequency splitting of the two $-C \equiv N$ stretching bands of $\mathbf{1}$ in the excited state as a function of the Onsager solvent polarity (red symbols) and best fit of the Ivanov symmetry-breaking model (solid green line). The grey zone represents the region below the threshold polarity where ES-SB is not operative. Solvent abbreviations: CHX: cyclohexane, DBE:

dibutyl ether; DEE: diethyl ether; THF: tetrahydrofuran; BCN: benzonitrile; DMSO: dimethyl sulfoxide; ACN: acetonitrile

Figure $5 \mathrm{~B}$ indicates that the splitting of these two $-C \equiv N$ bands increases with solvent polarity. We started collaborating on this topic with A. Ivanov during his visit in Geneva in 2016. He developed a theoretical model of ES-SB, where SB is favoured by the gain of dipolar solvation energy of the asymmetric state as well as by the reduced electrostatic repulsion of the partial charges on the two branches [13]. If the solvent is sufficiently polar, these two effects counterbalance the energy gained upon delocalisation of the excitation over the two branches. The threshold polarity above which ES-SB takes place depends on the electronic coupling between the branches. The solid green line in Figure $5 \mathrm{~B}$ is the best fit of this model to the experimentally observed band splitting. The agreement 
is excellent, the model predicts that ES-SB does not take place in the low polarity region represented in grey. The larger than predicted splitting observed in the weakly polar diethyl ether (DEE) can be accounted for by the quadrupolar nature of this solvent that is not included in the theory. This model was subsequently refined to better account for the effect of ES-SB on the IR spectrum [11].

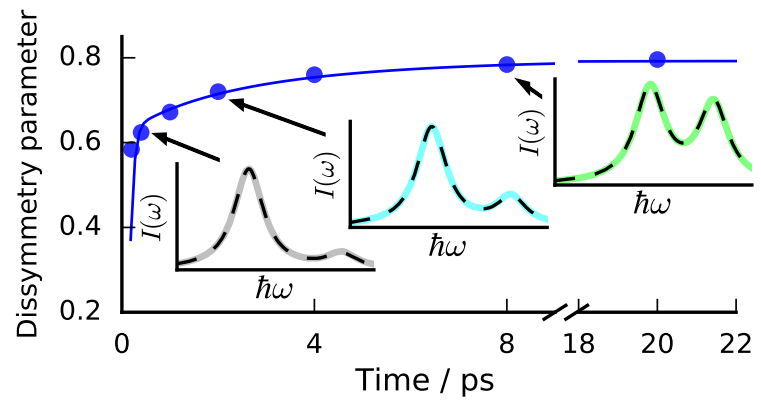

Fig. 6. Time dependence of the extent of ES-SB and of the transient IR spectrum in the $-C \equiv C-$ stretching region measured with dye $\mathbf{2}$ (Figure 4) in dimethyl formamide. The coloured and the dashed lines represent the experimental and calculated spectra, respectively. The symbols are the dissymmetry parameters extracted from the experimental spectra, whereas the solid blue line is its time dependence calculated from the model assuming a multiexponential dielectric response of the solvent (eprinted with permission from ref. 18, Copyright 2020, American Chemical Society)

Very recently, a significant extension of the model was realised, that allows describing the ES-SB dynamics and reproducing the temporal evolution of the IR spectra [18]. The spectral shape depends on the extent of SB, which is accounted for by a dissymmetry parameter, $D$. This model was applied to transient IR data measured in the $-C \equiv C-$ stretching region of dye $\mathbf{2}$ shown in Figure 4. The temporal evolution of $D$ and of the spectrum in solvent of varying polarity are very well reproduced assuming the ES-SB is controlled by solvation [18]. Figure 6 shows the time evolution of $D$ in the polar solvent DMF extracted from the analysis of the IR spectra together with that calculated from the model assuming a multiexponential dielectric response of the solvent. The parameters used to describe the solvent response were very similar to those measured experimentally. This figure also reveals that the IR spectral dynamics can be very well reproduced by the model. This excellent agreement between experiment and theory is an unambiguous evidence of the crucial role of the environment in the ES-SB process. It also indicates that structural fluctuations are not a prerequisite for ES-SB to take place.

In principle, such ES-SB process is not limited to quadrupolar two-branched molecules, but can also be operative in large conjugated systems. It can be viewed as a loss of coherence upon localisation of the exciton. A deep understanding of the factors that control this phenomenon is crucial for the development of new photoactive materials for various applications.

\section{Conclusions}

This long-standing collaboration between our experimental group in Geneva that the theory group of A. Ivanov in Volgograd has been very fruitful. It allowed deepening our understanding of the dynamics of charge-transfer processes in liquids that occur on similar 
timescale that those of vibrational and solvent relaxation. Although this is fundamental research, this knowledge is highly relevant for the numerous applications where these processes are involved. I am looking forward to further collaborative projects between our two groups in this exciting area.

\section{NOTE}

${ }^{1}$ Financial support from the Swiss National Science Foundation (grant 200020-184607) and the University of Geneva is acknowledged.

\section{REFERENCES}

1. Burshtein A.I. Non-Markovian Theories of Transfer Reactions in Luminescence and Chemiluminescence and Photo- and Electrochemistry. Adv. Chem. Phys., 2004, vol. 129, pp. 105-418.

2. Burshtein A.I. Unified Theory of Photochemical Charge Separation. Adv. Chem. Phys., 2000, vol. 114, pp. 419-587.

3. Demchenko A.P., Tomin V.I., Chou P.-T. Breaking the Kasha Rule for More Efficient Photochemistry. Chem. Rev., 2017, vol. 117, no. 21, pp. 13353-13381. DOI: 10.1021/acs.chemrev.7b00110.

4. Dereka B., Koch M., Vauthey E. Looking at Photoinduced Charge Transfer Processes in the IR: Answers to Several Long-Standing Questions. Acc. Chem. Res., 2017, vol. 50, no. 2, pp. 426-434. DOI: 10.1021/acs.accounts.6b00538.

5. Dereka B., Vauthey E. Solute-Solvent Interactions and Excited-State Symmetry Breaking: Beyond the Dipole-Dipole and the Hydrogen-Bond Interactions. J. Phys. Chem. Lett., 2017, vol. 8, pp. 3927-3932. DOI: 10.1021/acs.jpclett.7b01821.

6. Fedunov R.G., Feskov S.V., Ivanov A.I., Nicolet O., Pagès S., Vauthey E. Effect of the Excitation Pulse Carrier Frequency on the Ultrafast Charge Recombination Dynamics of Donor-Acceptor Complexes: Stochastic Simulations and Experiments. J. Chem. Phys., 2004, vol. 121, no. 8, pp. 3643-3656. DOI: http://dx.doi.org/10.1063/1.1772362.

7. Feskov S.V., Ionkin V.N., Ivanov A.I., Hagemann H., Vauthey E. Solvent and Spectral Effects in the Ultrafast Charge Recombination Dynamics of Excited DonorAcceptor Complexes. J. Phys. Chem. A, 2008, vol. 112, no. 4, pp. 594-601. DOI: http://dx.doi.org/10.1021/jp709587x.

8. Feskov S.V., Rogozina M.V., Ivanov A.I., Aster A., Koch M., Vauthey E. Magnetic Field Effect on Ion Pair Dynamics upon Bimolecular Photoinduced Electron Transfer in Solution. $J$. Chem. Phys., 2019, vol. 150, iss. 2, article ID: 024501. DOI: 10.1063/1.5064802.

9. Gladkikh V.S., Burshtein A.I., Feskov S.V., Ivanov A.I, Vauthey E. Hot Recombination of Photogenerated Ion Pairs. J. Chem. Phys., 2005, vol. 123, no. 24, article ID: 244510-11. DOI: http://dx.doi.org/10.1063/1.2140279.

10. Ionkin V.N., Ivanov A.I., Vauthey E. Charge Recombination in Electron Donor-Acceptor Complexes with Two Absorption Bands. Russ. J. Phys. Chem. A, 2009, vol. 83, no. 4, pp. 683688. DOI: http://dx.doi.org/10.1134/S0036024409040281.

11. Ivanov A.I. Theory of Vibrational Spectra of Excited Quadrupolar Molecules with Broken Symmetry. J. Phys. Chem. C, 1018, vol. 122, no. 51, pp. 29165-29172. DOI: 10.1021/acs.jpcc.8b10985.

12. Ivanov A.I., Belikeev F.N., Fedunov R.G., Vauthey E. The Effect of Excitation Pulse Carrier Frequency on Ultrafast Charge Recombination Dynamics of Excited DonorAcceptor Complexes. Chem. Phys. Lett., 2003, vol. 372, no. 1-2, pp. 73-81. DOI: http://dx.doi.org/10.1016/S0009-2614(03)00363-4. 
13. Ivanov A.I., Dereka B., Vauthey E. A Simple Model of Solvent-Induced SymmetryBreaking Charge Transfer in Excited Quadrupolar Molecules. J. Chem. Phys., 2017, vol. 146, no. 16, pp. 164306-164307. DOI: 10.1063/1.4982067.

14. Kumpulainen T., Lang B., Rosspeintner A., Vauthey E. Ultrafast Elementary Photochemical Processes of Organic Molecules in Liquid Solution. Chem. Rev., 2017, vol. 117, no. 16, pp. 10826-10939. DOI: http://dx.doi.org/10.1021/acs.chemrev.6b00491.

15. Marcus R.A., Sutin N. Electron Transfer in Chemistry and Biology. Biochim. Biophys. Acta, 1985, vol. 811, pp. 265-322.

16. Marcus R.A. On the Theory of Red.-Ox. Reactions Involving Electron Transfer. I. J. Chem. Phys., 1956, vol. 24, pp. 966-978.

17. Mikhailova V.A., Ivanov A.I., Vauthey E. Nonequilibrium Charge Recombination from the Excited Adiabatic State of Donor-Acceptor Complexes. J. Chem. Phys., 2004, vol. 121, iss. 13, pp. 6463-6469. DOI: https://doi.org/10.1063/1.1789940.

18. Nazarov A.E., Ivanov A.I., Vauthey E. Modelling IR Spectral Dynamics upon Symmetry Breaking of a Photo-Excited Quadrupolar Dye. J. Phys. Chem. C, 2020, vol. 124, pp. 2357-2369. DOI: 10.1021 /acs.jpcc.9b10565.

19. Nicolet O, Vauthey E. Ultrafast Nonequilibrium Charge Recombination Dynamics of Excited Donor-Acceptor Complexes. J. Phys. Chem. A, 2002, vol. 106, no. 23, pp. 5553-5562. DOI: $10.1021 / \mathrm{jp} 025542 \mathrm{c}$.

20. Rosspeintner A., Lang B., Vauthey E. Ultrafast Photochemistry in Liquids. Annu. Rev. Phys. Chem., 2013, vol. 64, no. 1, pp. 247-271. DOI: http://dx.doi.org/10.1146/annurevphyschem-040412-110146.

21. Terenziani F., Painelli A., Katan C., Charlot M., Blanchard-Desce M. Charge Instability in Quadrupolar Chromophores: Symmetry Breaking and Solvatochromism. J. Am. Chem. Soc., 2016, vol. 128, pp. 15742-15755.

22. Vauthey E. Photoinduced Symmetry-Breaking Charge Separation. ChemPhysChem, 2012, vol. 13, no. 8, pp. 2001-2011. DOI: 10.1002/cphc.201200106.

\section{ОБЪЕДИНЕНИЕ ТЕОРИИ И ЭКСПЕРИМЕНТА ДЛЯ ПОНИМАНИЯ ПРОЦЕССА СВЕРХБЫСТРОГО ФОТОИНДУЦИРОВАННОГО ПЕРЕНОСА ЗАРЯДА}

\section{Эрик Вотэ}

Доктор наук, профессор кафедры физической химии,

Женевский университет

eric.vauthey@unige.ch

набережная Эрнест-Ансермет, 30, СН-1211 г. Женева, Швейцария

Аннотация. В статье дается краткий обзор давнего сотрудничества между нашей экспериментальной группой по спектроскопии в Женеве и теоретической группой под руководством профессора А.И. Иванова в Волгограде. Это плодотворное сотрудничество привело к значительному расширению нашего понимания динамики нескольких сверхбыстрых процессов переноса заряда в конденсированной фазе.

Ключевые слова: разделение и рекомбинация зарядов, нарушение симметрии переносом электрона, спектроскопия с временным разрешением, теория переноса электрона, эффект магнитного поля. 